\title{
The choice of key geometric parameters in the numerical optimization of centrifugal compressor impellers
}

\author{
Vladimir Neverov ${ }^{1}$, Yuri Kozhukhov ${ }^{1, *}$, Sergey Kartashov ${ }^{1}$, and Vyacheslav Ivanov ${ }^{1}$ \\ ${ }^{1}$ Peter the Great St. Petersburg Polytechnic University, Polytechnicheskaya 29, St. Petersburg, \\ 195251, Russian Federation
}

\begin{abstract}
The article deals with the choice of key geometric parameters and the range of their variation in solving the optimization problem of centrifugal compressor impellers using computational fluid dynamics. The study was carried out using Numeca Fine / Turbo package. The influence of more than 10 geometric parameters on the efficiency and the head of the impeller was considered. The influence degree evaluation of investigated optimization parameters was provide by changing the parameters value in a preset range and analyzing their impact on the efficiency and head of the impeller. As a result, the main geometric parameters of optimization, which should be considered first, were identified. Other parameters may not be considered within the optimization problem, and can be assigned to the standard values. In addition, recommendations on optimal ranges of parameter values were given.
\end{abstract}

\section{Introduction}

Modern optimization algorithms together with mathematical models realized in packages of computational gas dynamics allow to automate the process of searching for optimal solutions $[4,5]$. The structure of the gas flow in the impellers of centrifugal compressors has a complex nature $[6,7]$, therefore numerical optimization of the flow makes it possible to obtain the most efficient impeller at low costs in comparison with field experiments.

However, when solving optimization problems, the question arises of the rationality and validity of the calculation models, criteria, parameters and optimization algorithms used [8]. Also, the efficiency of selecting the main optimization parameters and parametrization of the geometric model of the object under study is important.

The aim of work is to determine the main and minor geometric parameters of optimization of the impeller of the Central Committee, as well as to estimate the rational ranges of variation of the parameters considered.

The object of investigation is a high-pressure impeller of the first stage of an industrial air centrifugal compressor.

*Corresponding author: kozhukhov_yv@ mail.ru 


\section{Methods}

The computational part is performed using three-dimensional modeling of viscous flow using CFD methods in the Numeca Fine / Turbo software package. Information on the main aspects of the calculation in the complex used is given in [9].

An optimal calculation model has been chosen for the parameters of the grid model and the computational domain that provide the optimal relationship between the accuracy, the rate of convergence of the problem, and the time spent in calculating [10] for selected turbulence models Shear Stress Transport (SST) [11] and Spalart-Allmaras (SA ) [12].

The calculated region and shape of the impeller blades are shown in Fig. 1. The sample regions were not taken into account. Consequently, the values of efficiency on the graphs are polytropic hydraulic.
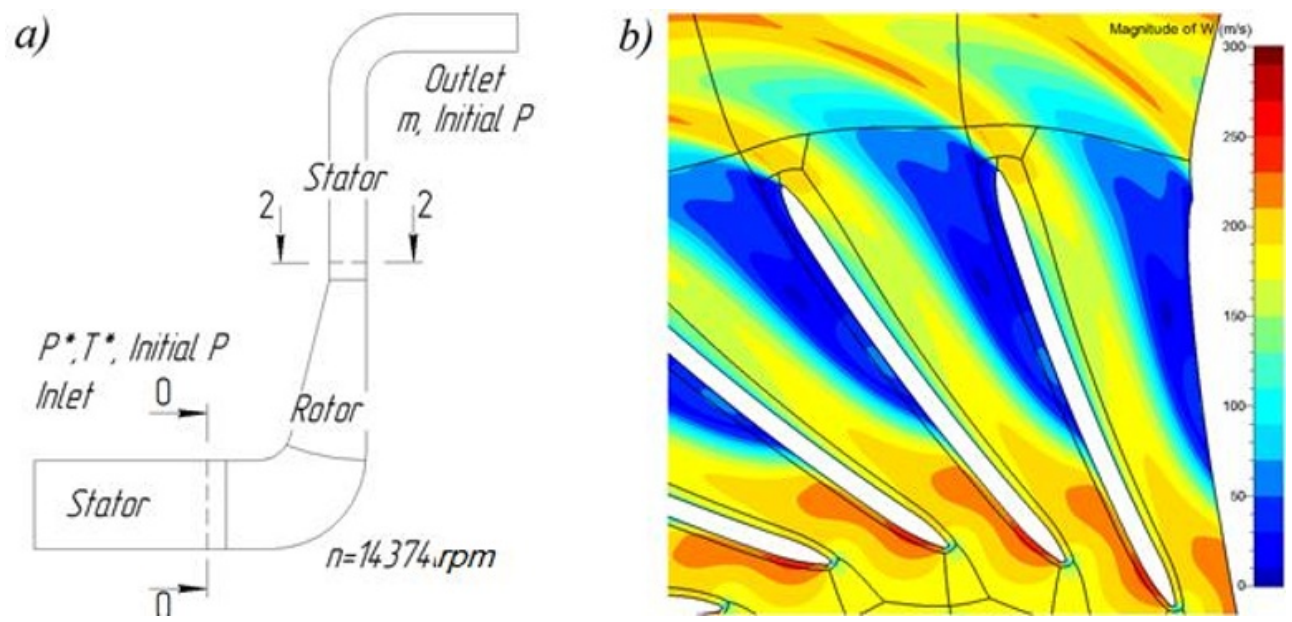

Fig. 1. Computational domain (a) and velocity contour plot at $50 \%$ span in the investigated impeller with the mean-camber curve of the blade formed by the circular arc (b)

\section{Results}

\subsection{The shape of the median blade line in the radial section}

The coefficients of the conjugate parabolas for the middle line of the wheel blades A and B varied in the ranges $\mathrm{A}=0.25 \ldots 0.35$ and $\mathrm{B}=0.2 \ldots 0.3$, recommended in the literature [13] (Figure 2), to search for the optimal combination. 

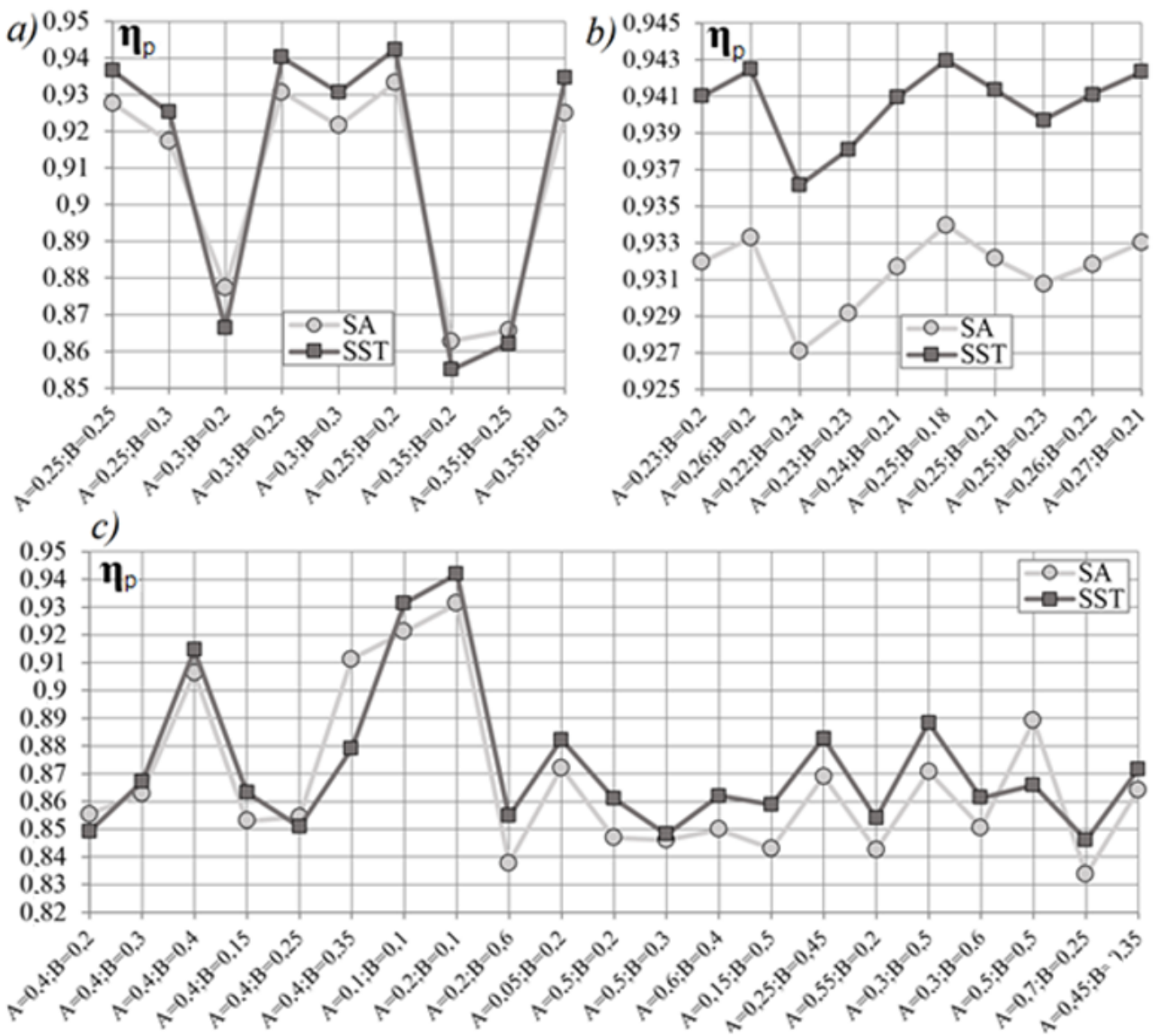

Fig. 2. Calculated values of the hydraulic efficiency at the design point for variations of coefficients $\mathrm{A}$ and $\mathrm{B}: \mathrm{a}$ ) in the range, which is recommended in literature [13]; b) in a small area of the best coefficients combination from (a); out of the range, which is recommended in literature [13]

For the given impeller, a design with combinations of coefficients greater than the recommended ones does not give acceptable efficiency.

\subsection{Meridian rounding of the cover and main disk}

The geometrical variation of the line of the covering disk is shown in Fig. 3. Table 1 shows the values of the dimensional $\mathrm{r}$ and the relative radius (referred to the diameter of the impeller $\mathrm{D}_{2}$ ). 


\section{EECE-2018}
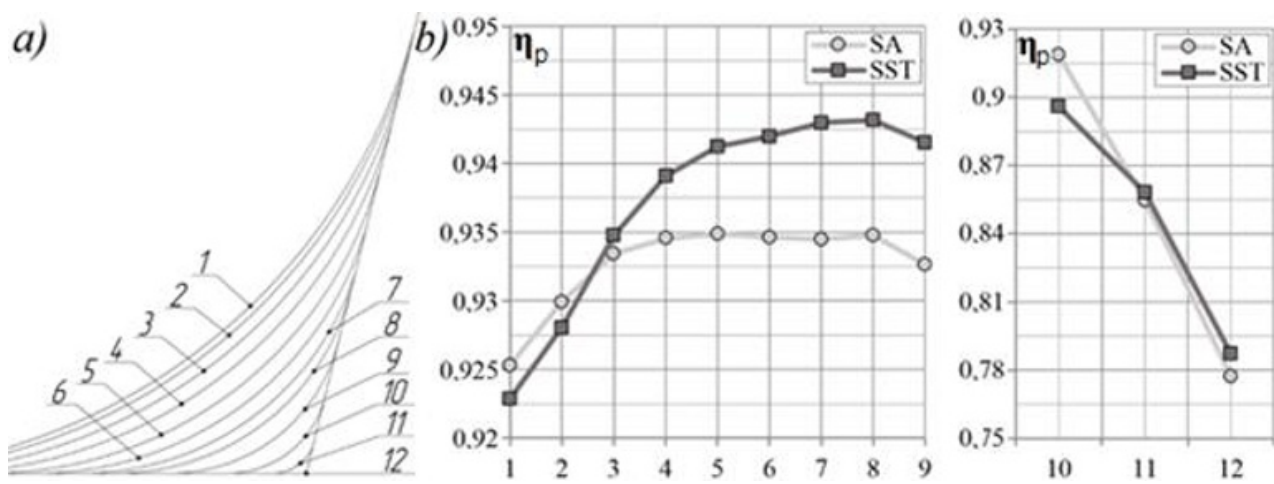

Fig. 3. Variations of the meridional shroud radius (a) and calculated values of the hydraulic efficiency at the design point (b)

Table 1. Variations of the meridional shroud radius

\begin{tabular}{|l|c|c|c|c|c|c|c|c|c|c|r|r|}
\hline № & 1 & 2 & 3 & 4 & 5 & 6 & 7 & 8 & 9 & 10 & 11 & 12 \\
\hline$r, \mathrm{~mm}$ & 182 & 174 & 160 & 143 & 122 & 104 & 87 & 73 & 52 & 30 & 13 & 0 \\
\hline $\bar{r}$ & 0.45 & 0.43 & 0.4 & 0.36 & 0.3 & 0.26 & 0.22 & 0.18 & 0.13 & 0.07 & 0.03 & 0 \\
\hline
\end{tabular}

The complex rounding of the covering disk, formed by a combination of several radii, straight lines and Bezier curves, is shown in Figure 4.
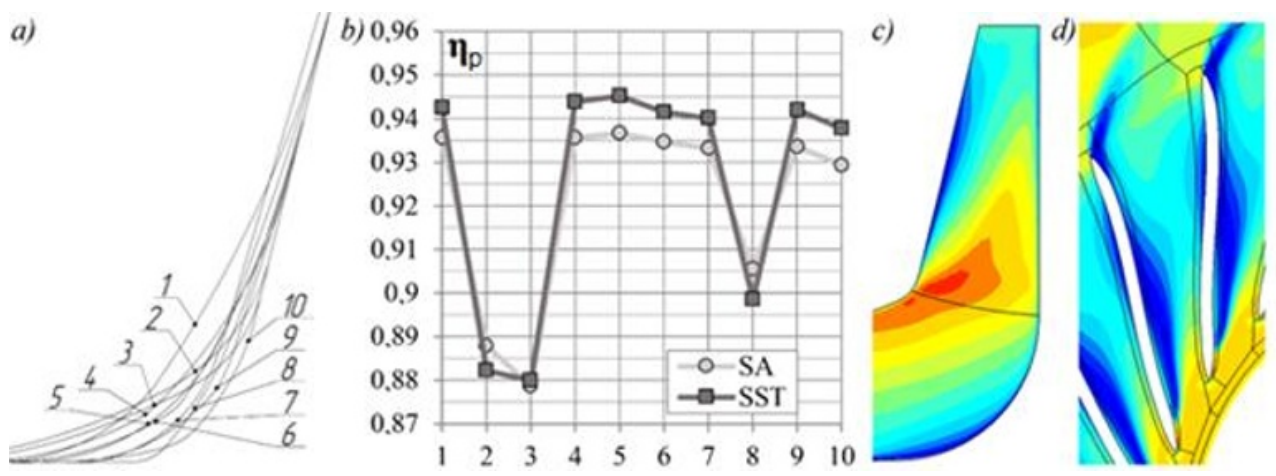

Fig. 4. Variations of the meridional shroud spline (a) and calculated values of the hydraulic efficiency at the design point (b); the meridional plot with the velocity contour for the variant №3 (c) and in the radial section at $95 \%$ span from the hub (d)

The variants of the rounding of the main disk are considered: the radius disk (Figure 5a) and the complex one (Figure 5c). Accordingly, Figures 5b and 5d show the calculated values of the hydraulic polytropic efficiency. Table 2 shows the values of the dimensional and relative radii for the radius transition of the meridional line of the main disk.

Table 2. Variations of the meridional hub radius

\begin{tabular}{|c|c|c|c|c|c|c|c|c|c|c|c|c|}
\hline № & 1 & 2 & 3 & 4 & 5 & 6 & 7 & 8 & 9 & 10 & 11 & 12 \\
\hline$r, \mathrm{~mm}$ & 152 & 134 & 121 & 109 & 95 & 85 & 78 & 67 & 53 & 41 & 29 & 19 \\
\hline $\bar{r}$ & 0.38 & 0.34 & 0.3 & 0.27 & 0.24 & 0.21 & 0.19 & 0.17 & 0.13 & 0.1 & 0.07 & 0.05 \\
\hline
\end{tabular}



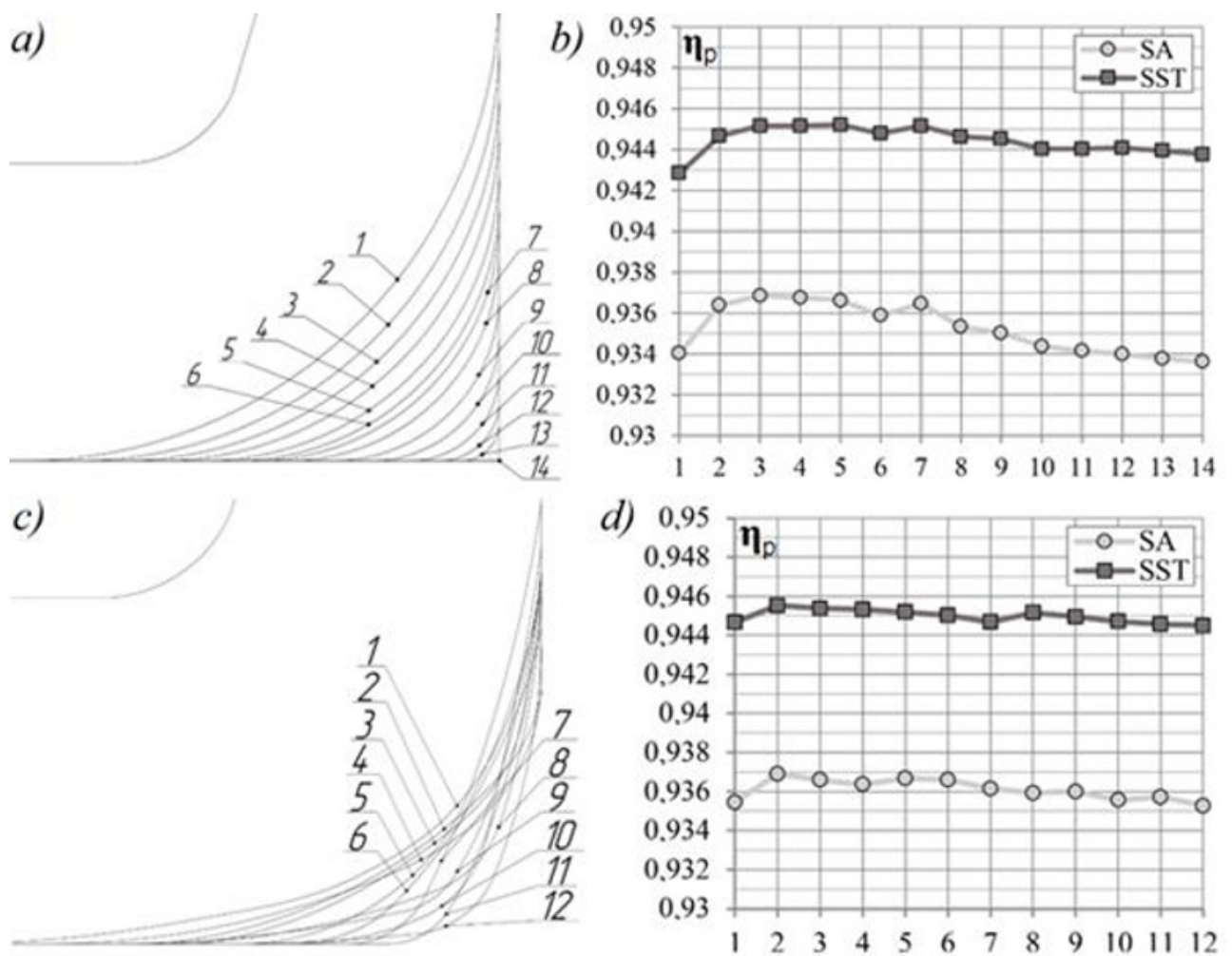

Fig. 5. Variations of the meridional hub radius and spline (a, b) and corresponding values of the hydraulic efficiency at the design point (c, d)

\subsection{The meridional shape of the covering disk within the interblade channel}

The variation of the meridian shape of the covering disk is shown in Fig. 6a, b.

The results of the calculation are shown in Fig. $6 \mathrm{c}$, d, e for 15 variants of the curved shape of the covering disk. 


\section{EECE-2018}
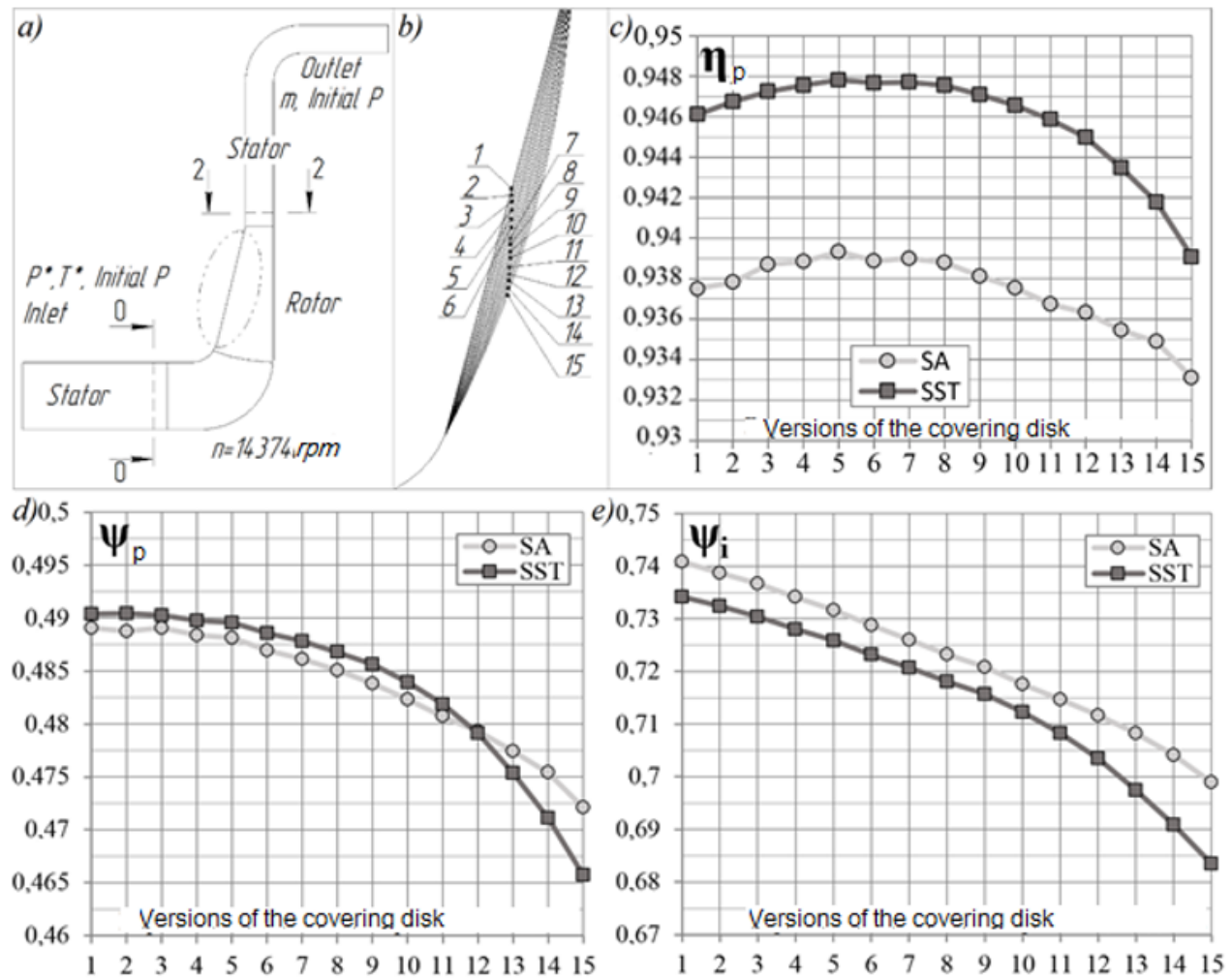

Fig. 6. Variation of the meridional shape of the impeller covering disk: a) Computational domain; b) variations of the impeller shroud; c) calculated values of the hydraulic efficiency; the polytropic (d) and total (e) head at the design point

The paper [14] compared the RC with the same load distribution on the blades with different meridional form, i.e. for different $b=f(r)$.

\subsection{Complex of parameters of the input edge in the meridional section}

The parameters of the input edge - diameter $D_{1}$, angle of inclination to the axis of rotation $\varphi$ and edge radius $\mathrm{R}$, determining the position and shape of the leading edge in the meridian plane (Fig. 7a, b, c) are considered separately. Variants with random combinations of these parameters are also calculated (Figure 7d), for which the calculation results are shown in Fig. 7e. 


\section{EECE-2018}

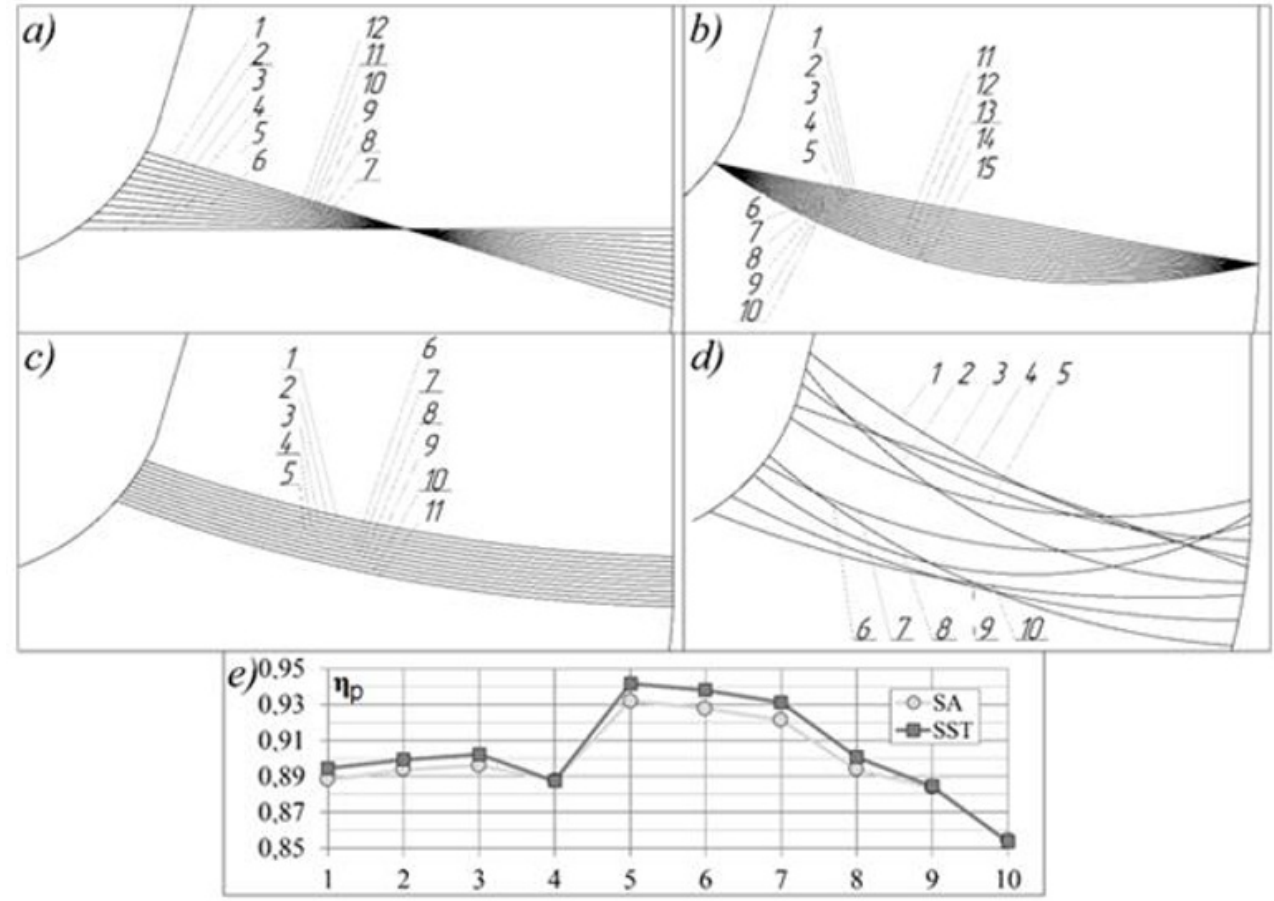

Fig. 7. Variation of the shape of the input edge of the impeller blades: a) variations of the meridional leading edge angle; $b$ ) the meridional radius shape of the leading edge; $c$ ) the leading edge diameter $\left.\mathrm{D}_{1} ; \mathrm{d}\right)$ a combination of leading edge parameters and corresponding calculated values of the hydraulic efficiency at the design point (e)

\subsection{Input and output edges in radial section}

The main variants of the input and output edges of the impeller blades are considered (Figure 8).

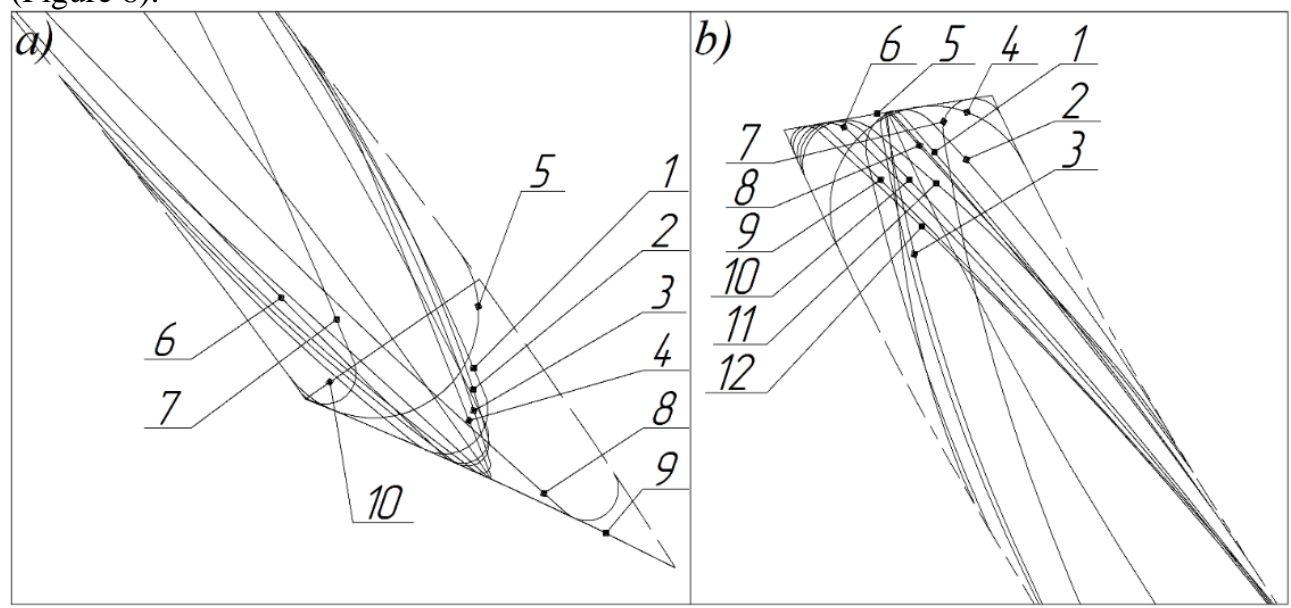

Fig. 8. Variations of the leading (a) and trailing (b) edges in the radial section Based on the results of the calculation, the input edges, made with a symmetrical fillet, show the best results. The overall effect of the shape of the output edge is small. 


\section{Discussion}

\subsection{Evaluation and selection of optimization parameters}

The influence of the bushing diameter, the diameter of the entrance to the $\mathrm{RC} \mathrm{D}_{0}$, the number of blades is considered.

As a result, all the parameters considered are summarized in Table 3. The contribution of the parameter was estimated both by the effect on the efficiency of the work of the impeller, and by the effect on the head. The value of the efficiency of the initial version of the impeller is $93.7 \%$.

Table 3. The influence degree evaluation of investigated optimization parameters on the impeller efficiency

\begin{tabular}{|l|c|l|}
\hline \multicolumn{1}{|c|}{$\begin{array}{c}\text { Parameter } \\
\text { Optimizing the Impeller }\end{array}$} & $\begin{array}{c}\text { efficiency } \\
\text { min, } \%\end{array}$ & \multicolumn{1}{|c|}{ Impact } \\
\hline The median blade line in the radial section & 86.3 & High \\
\hline Line covering the disk, radius & 77.7 & Average \\
\hline Line covering disk, polynomial & 87.9 & Average \\
\hline The main disk line, radius & 93.1 & Very Low \\
\hline The main disk line, the polynomial & 93.5 & Very Low \\
\hline $\begin{array}{l}\text { The complex of parameters is the generator, diameter, } \\
\text { inclination to the axial line of the input edge }\end{array}$ & 85.4 & High \\
\hline Input edge in the radial plane & 85.6 & Low * \\
\hline Output edge in the radial plane & 93.4 & Low \\
\hline Forming the covering disk within the interlop channel. & 93.3 & Low \\
\hline The input angle of the blade in the peripheral section & 93.8 & Low \\
\hline Sleeve diameter & 93.6 & - \\
\hline Diameter of the input to the impeller & 93.6 & Low \\
\hline
\end{tabular}

*-if you do not consider cropped edges.

\subsection{Conclusions}

It should be noted that, in addition to the analysis of geometric parameters, the selection of parameters of the calculation model, such as: the computational domain, grid parameters, boundary conditions, solver settings, etc., is of great importance in solving the optimization problem [15-20].

\section{References}

1. P. Le Sausse, P. Fabrie, D. Arnou, F. Clunet, EPJ Web. Conf. 45, 01059-P.1-8 (2013).

2. A.N. Arbekov, B.B. Novitskiy, Science and education. MGTU im. N. Uh. Bauman. Electron. Journal, 8, 491-501 (2012). DOI:10.7463/0812.0432308

3. O.V. Baturin, D.A. Kolmakova, V.N. Matveyev, Investigation of the centrifugal compressor working process using numerical methods of gas dynamics, 160 (2013)

4. F. Frese, J. Einzinger, J. Will, 10th International conference of turbocharges and turbocharging 2012, London, 121-134 (2012)

5. Y.V. Kozhukhov, A.M. Danilishin, V.K. Yun, IOP Conference Series Materials Science and Engineering 08/2015. 90(1), 012046 (2015). DOI:10.1088/1757-899X/90/1/012046

6. V.F. Ris, Mashinostroyeniye, p.351 (1981) 
7. K. P. Seleznev, Y. B. Galerkin. Mashinostroyeniye, p. 271 (1982)

8. V.L. Blinov, Yu.M. Brodov, V.A. Sedunin, O.V. Komarov. Compressor Engineering and Pneumatics, 1, 32-36 (2015).

9. O.V. Baturin, D.A. Kolmakova, V.N. Matveyev, G.M. Popov, L.S. Shabliy, The technique of digital modeling of axial multistage turbines of low pressure taking into account the uneven field of parameters at the entrance to the turbine, three-dimensional structure of the flow in the blade rings and leaks through the radial gaps of labyrinth seals, Samara (2012)

10. V.V. Neverov, Y.V. Kozhukhov, A.M. Yablokov, A.A. Lebedev, St. Petersburg State Polytechnic University Journal of Engineering Science and Technology, 4(254), 45-58 (2016) DOI:10.5862/JEST.254.6

11. P. R. Spalart, S. R. Allmaras. A One-Equation Turbulence Model for Aerodynamic Flows [Text]: conference, USA, (1992). doi: 10.2514/6.1992-439

12. F.R. Menter, AIAA Journal, 32(81994), 1598-1605. doi: 10.2514/3.12149

13. P. Seleznev, Y. B. Galerkin, B. N. Savin, Y. Y. Popova, R. A. Izmaylov, Design and optimization of the flow part of industrial centrifugal compressors using computers: tutorial, p. 76, (1990)

14. Y.B. Galerkin. Research, methods of calculation and design of the flow part of stationary centrifugal compressors. Abstract. dis. on competition of a scientific degree. scientist. tap dance. Doc. tech. sciences, p. 34 (1974)

15. L. Gibson, L. Galloway, S. Kim, S. Spence. Proceedings of the 1st Global Power and Propulsion Forum: GPPF 2017, Zurich, Switzerland, 16-18 January. (2017)

16. R. Aghaei Tog, A. M. Tousi, A. Tourani, Aircr. Eng. Aerosp. Tec., 80 (6), 657-665 (2008)

17. A.M. Karlov, A.F. Kuftov. Science and education. MGTU by. N. Uh. Bauman. Electron. Journal, 11, 69-80 (2012.) DOI:10.7463/1112.0465832.

18. F. Zhang, R. Baar. MATEC. Web of Conferences, 108, 08012 (2017)

19. D. E. van Zante, A. J. Strazisar, J.R. Wood, M.D. Hathaway, and T.D. Okiishi. Recommendations for achieving accurate numerical simulation of tip clearance flows in transonic compressor rotors. Paper TM2000-210347, NASA, (2000).

20. D.A. Roberts, R. Steed. A comparison of steady-state centrifugal stage CFD analysis to experimental rig data. 2004 ANSYS CFX conference, (2004). 